\title{
An efficient method for blind digital signal separation of array data
}

\author{
Cheng-Chou Lee, Ju-Hong Lee* \\ Room 517, Building 2, Department of Electrical Engineering, National Taiwan University, Taipei, 106, Taiwan
}

Received 27 May 1997; received in revised form 3 November 1998

\begin{abstract}
The paper deals with the problem of blind separation of multiple co-channel digital signals received by an antenna array. An efficient method based on the use of the symmetric property possessed by the received array data is proposed to significantly improve the performance of the approach previously presented in Anand et al. (1995). Moreover, the required computational complexity using the proposed method is reduced as compared to that using the conventional methods. The effectiveness of the proposed method is confirmed by computer simulation results. (C) 1999 Elsevier Science B.V. All rights reserved.
\end{abstract}

\section{Zusammenfassung}

Die Arbeit beschäftigt sich mit dem Problem der blinden Trennung mehrerer gleichkanaliger Digitalsignale, die von einer Antennengruppe empfangen werden. Eine effiziente Methode, die auf der Ausnutzung der Symmetrieeigenschaft der Antennenempfangsdaten beruht, wird vorgeschlagen, um die Leistungsfähigkeit des zuvor in Anand et al. (1995) vorgestellten Ansatzes deutlich zu verbessern. Darüber hinaus wird der benötigte Rechenaufwand beim Einsatz der vorgeschlagenen Methode im Vergleich zum Einsatz herkömmlicher Methoden verringert. Die Wirksamkeit der vorgeschlagenen Methode wird durch Computersimulationsergebnisse bestätigt. (C) 1999 Elsevier Science B.V. All rights reserved.

\section{Résumé}

Cet article traite du problème de la séparation aveugle de signaux numériques multiples sur un même canal, reçus par un réseau d'antennes. Une méthode efficace basée sur l'utilisation de la propriété de symétrie des données reçues par le réseau est proposée afin d'améliorer significativement les performances de l'approche présentée précédemment en Anand et al. (1995). De plus, la complexité de calcul requise par la méthode proposée est plus faible que celles des méthodes conventionnelles. L'effectivité de la méthode proposée est confirmée par des résultats de simulations sur ordinateur. (C) 1999 Elsevier Science B.V. All rights reserved.

Keywords: Blind signal separation; Co-channel signals

\footnotetext{
* Corresponding author.
} 


\section{Introduction}

Several techniques have been developed for solving the problem of separating the digital signals from the data of multiple co-channel digital signals plus noise received by an antenna array [1-3]. In this paper, we present an efficient method based on the exploitation of the symmetric property possessed by the data vectors received by an antenna array. In fact, the data vector $-\boldsymbol{x}(n)$ can be utilized whenever a data vector $\boldsymbol{x}(n)$ is received. Therefore, we can use the information embedded in both $\boldsymbol{x}(n)$ and $-\boldsymbol{x}(n)$ to improve the accuracy of the results during the clustering process. It is shown that the proposed method possesses the following two advantages over the approach presented in [2]. First, the number of the cluster sets required by the proposed method is reduced to half of that required by [2]. For example, we have to find only $2^{d-1}$ rather than $2^{d}$ cluster sets for $d$ users. This will reduce the computational complexity considerably especially when the number of users is large. Secondly, employing both $\boldsymbol{x}(n)$ and $-\boldsymbol{x}(n)$ results in the increase in the number of data vectors available for obtaining the cluster vectors during the clustering process. Therefore, the accuracy of the results obtained after clustering can be improved under the same input SNR. The effectiveness of the proposed method is confirmed by computer simulation results.

\section{The array data model and problem formulation}

Assume that $d$ narrowband digital signals are impinging on an antenna array with $m$ sensors from $d$ different users. The corresponding baseband data sampled at the $l$ th sensor can be expressed as [2]

$x_{l}(n)=\sum_{k=1}^{d} p_{k} a_{l k} b_{k}(n)+w_{l}(n)$,

where $p_{k}$ is the amplitude of the $k$ th user's signal, $a_{l k}$ is the response of the $l$ th sensor to the $k$ th user's signal, $b_{k}(n)$ represents the $n$th digital bit of the $k$ th user's signal, and $w_{l}(n)$ is the received white noise with variance $\sigma^{2}$. In vector form, (1) can be rewritten as

$\boldsymbol{x}(n)=\boldsymbol{A s}(n)+\boldsymbol{w}(n)$, where $\quad \boldsymbol{x}(n)=\left[x_{1}(n), \ldots, x_{m}(n)\right]^{\mathrm{T}}, \boldsymbol{s}(n)=\left[b_{1}(n), \ldots\right.$, $\left.b_{d}(n)\right]^{\mathrm{T}}, \quad \boldsymbol{w}(n)=\left[w_{1}(n), \ldots, w_{m}(n)\right]^{\mathrm{T}}, \quad$ and $\boldsymbol{A}=$ $\left[p_{1} \boldsymbol{a}_{1}, \ldots, p_{d} \boldsymbol{a}_{d}\right]$ with $\boldsymbol{a}_{k}=\left[a_{1 k}, \ldots, a_{m k}\right]^{\mathrm{T}}$. Taking $N$ data snapshots yields the following matrix representation:

$\boldsymbol{X}(N)=\boldsymbol{A S}(N)+\boldsymbol{W}(N)$,

where $\boldsymbol{X}(N)=[\boldsymbol{x}(1), \ldots, \boldsymbol{x}(N)], \quad \boldsymbol{S}(N)=[\boldsymbol{s}(1), \ldots$, $\boldsymbol{s}(N)]$, and $\boldsymbol{W}(N)=[\boldsymbol{w}(1), \ldots, \boldsymbol{w}(N)]$. The number $N$ is appropriately selected so that the array response matrix $\boldsymbol{A}$ varies negligibly over the $N$ baud periods $N T$. Based on (3), the problem of blind separation for multiple co-channel digital signals of array data in the presence of additive noise $\boldsymbol{w}(n)$ becomes a conventional estimation problem of the array response matrix $\boldsymbol{A}$ and the bit sequences contained in $\boldsymbol{S}(N)$, given the received array data $\boldsymbol{X}(N)$ [1-3].

For simplicity, consider the situation where all signals are modulated in the BPSK format. There are only $2^{d}$ possible distinct bit sequences for $s(n)$ in $\boldsymbol{S}(N)$. Assume that the array response matrix $\boldsymbol{A}$ is of full-column rank. Let all the $2^{d}$ possible distinct vectors $s(n)$ be the columns of the $d \times 2^{d}$ matrix $\boldsymbol{S}_{0}$ and $\boldsymbol{M}=\boldsymbol{A} \boldsymbol{S}_{0}$. Then, in the case of no additive noise $\boldsymbol{w}(n)$, it can be easily shown that $\boldsymbol{S}_{0} \boldsymbol{S}_{0}^{\mathrm{T}}=2^{d} \boldsymbol{I}_{d}$ and the columns of $\boldsymbol{M}$ are equal to the constellation vectors $\boldsymbol{x}(n)$, where $\boldsymbol{I}_{\boldsymbol{d}}$ denotes the identity matrix with size $d \times d$. Moreover, we have that $\boldsymbol{M M}^{\mathrm{T}}=$ $\boldsymbol{A} \boldsymbol{S}_{0} \boldsymbol{S}_{0}^{\mathrm{T}} \boldsymbol{A}^{\mathrm{T}}=2^{d} \boldsymbol{A} \boldsymbol{A}^{\mathrm{T}}$. Based on the above properties, the problem of blind separation for multiple co-channel digital signals of array data in the presence of additive noise $\boldsymbol{w}(n)$ can be viewed as the problem of clustering the received array data vectors in $\boldsymbol{X}(N)$ into $2^{d}$ cluster sets and assigning an appropriate bit sequence contained in $\boldsymbol{S}_{0}$ to each cluster set [2].

\section{The proposed method}

\subsection{Problem simplification using symmetric property}

Here, we exploit the symmetric property possessed by the received array data to simplify the problem described in Section 2. Consider the format in constructing all the possible bit sequences. We note that both the bit sequences represented by 
$\boldsymbol{s}(n)$ and $-\boldsymbol{s}(n)$ exist whenever one of them is generated. Hence, the matrix $\boldsymbol{S}_{0}$ containing all the $2^{d}$ possible distinct vectors $\boldsymbol{s}(n)$ can be partitioned as follows:

$\boldsymbol{S}_{0}=\left[\hat{\boldsymbol{S}}_{0}-\hat{\boldsymbol{S}}_{0}\right]=\hat{\boldsymbol{S}}_{0} \boldsymbol{J}$,

where $\boldsymbol{J}=\left[\boldsymbol{I}_{L},-\boldsymbol{I}_{L}\right]$ and $\boldsymbol{I}_{L}$ denotes the identity matrix with size $L=2^{d-1}$. Accordingly, the matrix $\boldsymbol{M}$ containing the constellation vectors can be expressed as follows:

$\boldsymbol{M}=\boldsymbol{A} \boldsymbol{S}_{0}=\hat{\boldsymbol{M}} \boldsymbol{J}$,

where $\hat{\boldsymbol{M}}=\boldsymbol{A} \widehat{\boldsymbol{S}}_{0}$. Based on (5), it is easy to show that

$\hat{M} \hat{M}^{\mathrm{T}}=L A A^{\mathrm{T}}$.

Eq. (6) reveals that the number of the constellation vectors required for estimating the bit sequences reduces to $2^{d-1}$ instead of $2^{d}$. The computational complexity required for performing blind separation of the digital signals can therefore be alleviated.

\subsection{The clustering algorithm}

Basically, the considered problem as described in Section 3.1 can be viewed as a clustering problem in which the array response matrix $\boldsymbol{A}$ maps the source constellation associated with $\hat{\boldsymbol{S}}_{0}$ onto the transformed constellation associated with $\hat{\boldsymbol{M}}$. In the following, we therefore present a clustering algorithm based on the algorithm presented in [2] to perform the classification of the received $m \times 1$ data vectors $\boldsymbol{x}(n)$ for $n=1,2, \ldots, N$ into $L$ index clusters $\mathscr{F}^{(l)}, l=1,2, \ldots, L$.

Let the cluster vector $c^{(l)}$ be the mean of the cluster vectors associated with the index cluster $\mathscr{F}^{(l)}$ and the Euclidean distance in the space $C^{m}$ be denoted as $\operatorname{dist}(\boldsymbol{a}, \boldsymbol{b})$ for any two constellation vectors $\boldsymbol{a}, \boldsymbol{b} \in C^{m}$. From the result obtained in Section 3.1 , we note that for a given constellation vector $\boldsymbol{x}(n),-\boldsymbol{x}(n)$ is also a constellation vector when $\boldsymbol{w}(\boldsymbol{n})$ is absent. As a result, under the same clustering operation, using both $\boldsymbol{x}(n)$ and $-\boldsymbol{x}(n)$ produces exactly the same index clusters and cluster vectors as using only $\boldsymbol{x}(n)$. Therefore, in presence of noise, the data vectors $-\boldsymbol{x}(n)$ can be utilized during the clustering operation to equivalently increase the number of data snapshots and, hence, improve the accuracy of the results after clustering operation. The proposed clustering algorithm is summarized as follows:

Step 0 (Initialization). For $l=1,2, \ldots, L$, let $\mathscr{F}^{(l)}=\{l\}, n^{(l)}=1$, and $\boldsymbol{c}^{(l)}=\boldsymbol{x}(l)$,

Next, $r \leftarrow L+1$, where the notation " $r \leftarrow L+1$ " denotes that we set $r$ equal to $L+1$.

Step 1. For $i=1,2, \ldots, 2^{d}$, compute the distances $d_{x c}(r, i)$ as follows:

$d_{x c}(r, i)=\operatorname{dist}\left\{(-1)^{i+1} \boldsymbol{x}(r), \boldsymbol{c}^{\lfloor(i+1) / 2\rfloor}\right\}$,

where $\lfloor a\rfloor$ denotes the nearest integer not greater than $a$. Let

$i^{*} \leftarrow \arg \min _{i \in\left(1, \ldots, 2^{d}\right)} d_{x c}(r, i)$ and $l^{*}=\left\lfloor\left(i^{*}+1\right) / 2\right\rfloor$.

Next, for $l=1,2, \ldots, L$ and $i=1,2, \ldots, 2^{d}$, compute the distances $d_{c c}(l, i)$ as follows:

$$
\begin{aligned}
& d_{c c}(l, i)=\operatorname{dist}\left\{c^{(l)},(-1)^{i+1} c^{\lfloor(i+1) / 2\rfloor}\right\} \\
& \quad \text { if }\lfloor(i+1) / 2\rfloor \neq l, \\
& d_{c c}(l, i)=\infty \text { if }\lfloor(i+1) / 2\rfloor=l,
\end{aligned}
$$

where $\infty$ denotes the positive infinity. Let

$$
\left(l_{1}, i_{2}\right) \leftarrow \arg \min _{i \in(1, \ldots, L), i \in\left(1, \ldots, 2^{d}\right)} d_{c c}(l, i) .
$$

Step 2. If $d_{x c}\left(r, i^{*}\right)<d_{c c}\left(l_{1}, i_{2}\right)$, then

$$
\begin{aligned}
& \mathscr{F}\left(l^{*}\right) \leftarrow \mathscr{F}\left(l^{*}\right) \cup\left\{(-1)^{i^{*}+1} r\right\} \\
& c^{\left(l^{*}\right)} \leftarrow \frac{n^{\left(l^{*}\right)} c^{\left(l^{*}\right)}+(-1)^{i^{*}+1} \boldsymbol{x}(r)}{n^{\left(l^{*}\right)}+1} \\
& n^{\left(l^{*}\right)} \leftarrow n^{\left(l^{*}\right)}+1 .
\end{aligned}
$$

Otherwise,

$$
\begin{aligned}
& l_{2}=\left\lfloor\left(i_{2}+1\right) / 2\right\rfloor \\
& \mathscr{F}^{\left(l_{1}\right)} \leftarrow \mathscr{F}\left(l_{1}\right) \cup(-1)^{i_{2}+1} \mathscr{F}^{\left(l_{2}\right)} \\
& \mathscr{F}^{\left(l_{2}\right)} \leftarrow\left\{(-1)^{i^{*}+1} r\right\} \\
& \boldsymbol{c}^{\left(l_{1}\right)} \leftarrow \frac{n^{\left(l_{1}\right)} \boldsymbol{c}^{\left(l_{1}\right)}+(-1)^{i_{2}+1} n^{\left(l_{2}\right)} \boldsymbol{c}^{\left(l_{2}\right)}}{n^{\left(l_{1}\right)}+n^{\left(l_{2}\right)}} \\
& \boldsymbol{c}^{\left(l_{2}\right)} \leftarrow(-1)^{i^{*}+1} \boldsymbol{x}(r) \\
& n^{\left(l_{1}\right)} \leftarrow n^{\left(l_{1}\right)}+n^{\left(l_{2}\right)} \\
& n^{\left(l_{2}\right)} \leftarrow n^{\left(l_{2}\right)}+1
\end{aligned}
$$


Step 3. $r \leftarrow r+1$. If $r \leqslant N$, then go to Step 1. Otherwise, we terminate the process.

From the above algorithm, we note that the number of the cluster sets based on the proposed method is only half of that based on the approach of [2]. The notation $(-1)^{i_{2}+1} \mathscr{F}\left(l_{2}\right)$ used in Step 2 denotes the set whose entries are equal to $(-1)^{i_{2}+1}$ times the entries of $\mathscr{F}\left(l_{2}\right)$. Moreover, the data vector $\boldsymbol{x}(r)$ is mapped to the cluster vector $-c^{(l)}$ if the index $-r$ is contained in $\mathscr{F}^{(l)}$.

\subsection{The assignment algorithm}

After obtaining the cluster vectors $c^{(l)}$, we construct the matrix $\hat{\boldsymbol{C}}=\left[c^{(1)}, \ldots, c^{(L)}\right]$. Based on the symmetric property exploited in Section 3.1, the matrix containing all possible cluster vectors is given by $\boldsymbol{C}=\hat{\boldsymbol{C}} \boldsymbol{J}$. Next, consider the SVD of $\hat{\boldsymbol{C}}$. Let $\widehat{U}_{m \times d} \widehat{\Sigma}_{d \times d} \hat{V}_{d \times L}$ be the truncated SVD of $\hat{\boldsymbol{C}}$, then the corresponding truncated SVD of $\boldsymbol{C}$ is given by $\hat{U}(\sqrt{2} \hat{\Sigma})\left(\sqrt{2}^{-1} \hat{\boldsymbol{V}} \boldsymbol{J}\right)$. Using this property, we present an appropriate algorithm for assigning the cluster vectors obtained from Section 3.2 to the bit sequences contained in the matrix $\boldsymbol{S}_{0}$. The proposed assignment algorithm is summarized as follows:

Step 1. Perform the truncated SVD of $\hat{\boldsymbol{C}}=$ $\widehat{U}_{m \times d} \hat{\Sigma}_{d \times d} \hat{V}_{d \times L}$.

Step 2. Assign the column vector $e_{1}=[1,1, \ldots, 1]^{\mathrm{T}}$ as the first column of $\hat{\boldsymbol{S}}_{0}$.

Step 3. Compute the row vector $\boldsymbol{q}=L \boldsymbol{v}_{1}^{\mathrm{T}} \hat{\boldsymbol{V}} \boldsymbol{J}$, where $v_{1}$ is the first column vector of $\hat{\boldsymbol{V}}$.

Step 4. Find the indices $i_{j}$ such that the entries $q\left(i_{j}\right)$ of $\boldsymbol{q}$ for $j=1,2, \ldots, d-1$ are closest to $d-2$.

Step 5. Assign the column vectors $\boldsymbol{e}_{2}=$ $[-1,1, \ldots, 1]^{\mathrm{T}}, \boldsymbol{e}_{3}=[1,-1, \ldots, 1]^{\mathrm{T}}, \ldots, \boldsymbol{e}_{d}=[1$, $\cdots,-1,1]^{\mathrm{T}}$ as the columns $i_{1}, i_{2}, \ldots, i_{d-1}$ of $\hat{\boldsymbol{S}}_{0} \boldsymbol{J}$, and the column vectors $v_{i_{1}} \boldsymbol{v}_{i_{2}} \ldots \boldsymbol{v}_{i_{d-1}}$ as the columns $i_{1}, i_{2}, \ldots, i_{d-1}$ of $\hat{\boldsymbol{V}} \boldsymbol{J}$.

Step 6. Construct the matrix $\boldsymbol{P}=\tilde{\boldsymbol{V}} \boldsymbol{E}^{-1} \sqrt{L}$, where $\tilde{V}=\left[\begin{array}{lllll}v_{1} & v_{i_{1}} & v_{i_{2}} & \ldots & v_{i_{d-1}}\end{array}\right]$ and $E=\left[\begin{array}{lll}e_{1} & e_{2} \ldots & e_{d}\end{array}\right]$.

Step 7. Construct the matrix $\hat{\boldsymbol{S}}_{0}=\boldsymbol{P}^{-1} \hat{\boldsymbol{V}} \sqrt{L}$.

Step 8: Let $\boldsymbol{s}_{l}$ be the lth column of $\hat{\boldsymbol{S}}_{0}$ for $l=1, \ldots, L$, If $(-1)^{i} r$ is contained in $\mathscr{F}^{(l)}$, then the bit sequence corresponding to $\boldsymbol{x}(r)$ is given by $(-1)^{i} s_{l}$.
We note that only the SVD of an $m \times 2^{d-1}$ matrix rather than an $m \times 2^{d}$ matrix must be performed during the proposed assignment algorithm. Therefore, the proposed method reduces the required computational burden considerably as compared to the approach presented in [2].

\section{Computer simulation results}

In this section, several simulation results are presented for illustration and comparison. For all simulations, both the proposed method and the approach presented in [2] are performed. The array used is a uniform linear array with $m=5$ and the interelement spacing is equal to half the signal wavelength. Three BPSK signals are equi-powered and impinging on the array from $\left[-10^{\circ}, 3^{\circ}, 12^{\circ}\right]$ off broadside. Moreover, the number of snapshots used is $N=50$. Each entry of the matrix $\boldsymbol{S}(N)$ takes the value of 1 or -1 randomly. All the simulation results are obtained by averaging over 2000 Monte Carlo trials for each SNR.

Example 1. In this example, we consider the case of fixed initial phase for the three digital signals, i.e., the vector $\boldsymbol{a}_{k}$ in (2) is set to

$\boldsymbol{a}_{k}=\left[1, \mathrm{e}^{\mathrm{j} \pi \sin \left(\theta_{k}\right)}, \ldots, \mathrm{e}^{\mathrm{j}(m-1) \pi \sin \left(\theta_{k}\right)}\right]^{\mathrm{T}}$.

Accordingly, the array response matrix $\boldsymbol{A}$ is fixed during the 2000 Monte Carlo trials. Table 1 lists the simulation results in terms of the BER for different SNRs and users.

Example 2. Here, the case of the three digital signals with random initial phase is considered. We let the vector $\boldsymbol{a}_{k}$ in (2) be given by

$\boldsymbol{a}_{k}=\mathrm{e}^{\mathrm{j} \pi \alpha_{k}}\left[1, \mathrm{e}^{\mathrm{j} \pi \sin \left(\theta_{k}\right)}, \ldots, \mathrm{e}^{\mathrm{j}(m-1) \pi \sin \left(\theta_{k}\right)}\right]^{\mathrm{T}}$,

where $\alpha_{k}$ is a random variable uniformly distributed in the interval $[-1,1]$. During each Monte Carlo trial, we choose $\alpha_{k}$ randomly for $k=1,2, \ldots, d$. Table 2 shows the simulation results in terms of the BER for different SNRs and users.

From the simulation results shown by the tables, we observe that the proposed method significantly outperforms the approach presented in [2]. 
Table 1

Bit error rate $(B E R) \times 10^{3}$ versus different SNRs for fixed signal initial phases

SNR in $\mathrm{dB}$

\begin{tabular}{lllllllllllll}
\hline 0 & 1 & 2 & 3 & 4 & 5 & 6 & 7 & 8 & 9 & 10
\end{tabular}

(a) Using the approach of [2]

\begin{tabular}{|c|c|c|c|c|c|c|c|c|c|c|c|}
\hline BER for user1 & 52.60 & 27.07 & 12.81 & 4.92 & 2.59 & 1.56 & 1.15 & 0.79 & 0.27 & 0.00 & 0.00 \\
\hline BER for user2 & 126.34 & 63.01 & 36.73 & 19.12 & 10.17 & 5.86 & 2.33 & 1.09 & 1.13 & 0.25 & 0.24 \\
\hline BER for user3 & 83.66 & 39.48 & 19.77 & 10.27 & 4.08 & 3.04 & 1.44 & 0.47 & 0.33 & 0.20 & 0.00 \\
\hline \multicolumn{12}{|c|}{ (b) Using the proposed method } \\
\hline BER for user1 & 22.65 & 4.86 & 1.23 & 0.46 & 0.01 & 0.00 & 0.00 & 0.00 & 0.00 & 0.00 & 0.00 \\
\hline BER for user2 & 42.67 & 13.63 & 3.59 & 0.86 & 0.44 & 0.10 & 0.00 & 0.00 & 0.00 & 0.00 & 0.00 \\
\hline BER for user3 & 32.43 & 7.70 & 1.30 & 0.13 & 0.04 & 0.00 & 0.00 & 0.00 & 0.00 & 0.00 & 0.00 \\
\hline
\end{tabular}

Table 2

Bit error rate $(B E R) \times 10^{3}$ versus different SNRs for random signal initial phases

\section{SNR in $\mathrm{dB}$}

1

(a) Using the Approach of [2]

$\begin{array}{lrrr}\text { BER for user1 } & 65.65 & 34.45 & 12.90 \\ \text { BER for user2 } & 169.07 & 101.57 & 53.96\end{array}$

$\begin{array}{lrrr}\text { BER for user3 } & 133.86 & 82.01 & 38.62\end{array}$

$\begin{array}{rr}8.49 & 3.41 \\ 27.86 & 14.20 \\ 19.89 & 7.58\end{array}$

$\begin{array}{ll}1.90 & 0.94 \\ 5.31 & 2.83 \\ 2.62 & 1.36\end{array}$

0.36
1.21
0.25

$\begin{array}{lll}0.22 & 0.25 & 0.19 \\ 1.12 & 0.65 & 0.13 \\ 0.86 & 0.33 & 0.00\end{array}$

(b) Using the proposed method

\begin{tabular}{llrrrrrrrrrr} 
BER for user1 & 27.03 & 6.97 & 1.24 & 0.70 & 0.06 & 0.03 & 0.00 & 0.00 & 0.00 & 0.00 & 0.00 \\
BER for user2 & 91.90 & 44.53 & 16.97 & 4.81 & 1.29 & 0.15 & 0.03 & 0.01 & 0.00 & 0.00 & 0.00 \\
BER for user3 & 81.16 & 40.54 & 15.90 & 4.59 & 1.08 & 0.15 & 0.03 & 0.01 & 0.00 & 0.00 & 0.00 \\
\hline
\end{tabular}

\section{Conclusion}

This paper has presented an efficient method for the problem of blind separation of multiple cochannel digital signals received by an antenna array. We have exploited the symmetric property possessed by the received array data to significantly improve the performance of the approach previously presented in [2]. Moreover, the required computational complexity using the proposed method can be reduced as compared to that using the conventional approaches. The effectiveness of the proposed method has been confirmed by computer simulation results performed by using BPSK signals. For other modulation formats like QPSK where the similar symmetric property is also possessed by the data received at an antenna array, the proposed method is expected to have the advantages of much better performance and less computational burden over the conventional approaches.

\section{Acknowledgements}

This work was supported by the National Science Council under Grant NSC86-2213-E002-054. 


\section{References}

[1] Alle-Jan van der Veen, Analytical method for blind binary signal separation, IEEE Trans. Signal Process. 45 (April 1997) 1078-1082.

[2] K. Anand, G. Mathew, V.U. Reddy, Blind separation of multiple co-channel BPSK signals arriving at an antenna array, IEEE Signal Process. Lett. 2 (September 1995) 176-178.
[3] S. Talwar, M. Viberg, A. Paulraj, Blind estimation of synchronous co-channel digital signals using an antenna array. Part I: algorithms, IEEE Trans. Signal Process. 44 (May 1996) 1184-1197. 